\title{
Metabolic emergencies in pregnancy
}

\author{
Authors: Naina Mohan ${ }^{A}$ and Anita Banerjee ${ }^{B}$
}

In pregnancy, women are more likely to develop certain metabolic disturbances as a result of the physiological changes that occur. Diabetic ketoacidosis and hypoglycaemia occur at increased frequency in women with pre-existing and gestational diabetes, and starvation ketoacidosis can present towards the end of pregnancy and can cause severe illness. Peripartum hyponatraemia is increasingly recognised and can be associated with maternal and neonatal morbidity. This review describes these conditions in detail as well as treatment priorities and the impact on both mother and baby.

\section{Introduction}

Physiological changes in pregnancy can lead to a predisposition to severe metabolic disturbance (summarised in Table 1). ${ }^{1}$ Insulin sensitivity initially increases in the first trimester but then decreases thereafter to a nadir of approximately $56 \%$ by the end of the third trimester. ${ }^{2}$ This is partially driven by placentallyderived hormones, such as cortisol, glucagon and human placental lactogen. Pregnant women also have a lowered renal buffering capacity as their increased minute ventilation causes a respiratory alkalosis and renal bicarbonate loss, making them particularly prone to acidosis.

\section{Diabetic ketoacidosis}

Diabetic ketoacidosis (DKA) can occur in pregnant women with pre-existing or gestational diabetes mellitus. DKA in pregnancy can develop very rapidly and at lower blood glucose concentrations than in the non-pregnant population. Euglycaemic DKA is also seen in pregnancy. ${ }^{3}$ All women with type 1 diabetes mellitus (T1DM) are given a blood ketone meter for use at home, and are asked to seek medical attention if readings are above $2 \mathrm{mmol} / \mathrm{L}$.

DKA remains uncommon, with a reported incidence of $0.5 \%-3 \%{ }^{4}$ The perinatal mortality rate is between $9 \%-36 \%$ with a maternal mortality rate of less than $1 \% .{ }^{5}$ Risk factors include nausea and vomiting of pregnancy, concurrent infections, and poor compliance with medications or steroid therapy (particularly intramuscular steroids given for fetal lung maturation). The treatment of DKA in

Authors: ${ }^{\mathrm{A}}$ senior clinical fellow in obstetric medicine and specialist registrar in acute internal medicine, St Thomas' Hospital, London, UK; ${ }^{B}$ consultant obstetric physician, diabetologist and endocrinologist, St Thomas' Hospital, London, UK

\begin{tabular}{ll} 
Table 1. Metabolic changes in normal pregnancy \\
\hline Fasting glucose levels & Decreased \\
Glucose tolerance & Decreased \\
Insulin sensitivity & Decreased \\
Insulin production & 2 -fold increase \\
Maternal adipose tissue & Increased \\
Total triglycerides & $2-4$-fold increase \\
Total cholesterol & $0.25-0.4$-fold increase
\end{tabular}

pregnancy is the same as in non-pregnant populations: intravenous fluid resuscitation with normal saline, with the addition of glucose depending on serum glucose levels, intravenous insulin delivered at a fixed rate and electrolyte replacement.

Delivery is not indicated as treatment for DKA, but there is significant fetal mortality associated with severe DKA and so appropriate fetal monitoring based on gestational age should be instituted. Delivery may be required when the mother has been

\section{Key points}

Hypoglycaemia is more common in women with preexisting diabetes when pregnant, and is treated as in the non-pregnant population.

Diabetic ketoacidosis in pregnant women can occur rapidly and at lower blood glucose concentrations than in nonpregnant individuals.

Starvation ketoacidosis can occur in the third trimester in association with a short duration of vomiting or reduced oral intake. Treatment is with intravenous glucose.

Hyponatraemia is common in labour, often related to excessive fluid intake in combination with the antidiuretic hormone-like effects of high oxytocin levels.

The treatment of hyponatraemia in labour is dependent on the cause. Hypertonic sodium chloride is reserved for symptomatic cases of severe hyponatraemia.

KEYWORDS: pregnancy, ketoacidosis, starvation, diabetes, hyponatraemia

DOI: 10.7861/clinmed.2021-0496 
stabilised if severe fetal compromise is identified that does not respond to maternal resuscitation and medical treatment.

\section{Hypoglycaemia}

Hypoglycaemia is more common in pregnant women with preexisting diabetes, particularly in the first trimester. Risk factors include severe hypoglycaemic episodes in the previous year, long duration of diabetes mellitus and a glycated haemoglobin $\left(\mathrm{HbA}_{1 c}\right)$ concentration less than or equal to $6.5 \%$ in early pregnancy. Women may lose their hypoglycaemia awareness in pregnancy. The aim of achieving optimal glycaemic control may result in women reducing their hyperglycaemia but this may increase the risk of hypoglycaemia. The new National Institute for Health and Care Excellence (NICE) guidelines recommend continuous glucose monitoring to help women meet their pregnancy glucose targets and improve neonatal outcomes, but also can allow early recognition of hypoglycaemic episodes. ${ }^{6}$

All pregnant women on insulin should be advised to carry simple carbohydrates with them at all times, and pregnant women with T1DM are provided with emergency intramuscular glucagon. Their close contacts should be trained in the symptoms and signs to be aware of and how to deliver the glucagon, if required. Despite this, more than one in 10 pregnant women with T1DM are admitted to hospital with hypoglycaemia. ${ }^{7}$ Treatment includes oral glucose, followed by intravenous glucose administration or intramuscular glucagon if needed.

\section{Starvation ketoacidosis}

In the healthy adult population, prolonged starvation can lead to a mild ketoacidosis, but this can take 2 weeks to develop. Ketone levels are only mildly elevated and arterial $\mathrm{pH}$ is generally above $7.3 .^{8}$ This process is markedly accelerated in pregnancy, particularly in the second and third trimesters. Insulin resistance increases as pregnancy progresses and this, coupled with starvation and depleted glycogen stores, may lead to ketonaemia as fatty acids are metabolised for the citric acid cycle rather than glycogen.

Ketosis is relatively common in asymptomatic pregnant women, particularly first thing in the morning or following prolonged labour. There is no evidence of adverse outcomes if there is no dehydration or acidosis. ${ }^{9}$ However, women can present with starvation ketoacidosis, particularly following vomiting or reduced oral intake in the third trimester. These women are unwell with tachypnoea, tachycardia, a severe acidosis (pH less 7.3, low bicarbonate or significant base deficit), ketonaemia above $1 \mathrm{mmol} / \mathrm{L}$ and a blood glucose in or below the normal range, although significant respiratory compensation may be evident initially. ${ }^{9}$

Treatment involves intravenous glucose (for example, 10\%-20\% in strength), alongside other fluids required for resuscitation. If ketones remain high after initial resuscitation, a fixed rate of intravenous insulin can also be used. As in women with DKA, delivery of the fetus is usually not required, but fetal assessment should be performed urgently and delivery considered if fetal compromise is evident despite maternal resuscitation and treatment. Importantly, the cause for the vomiting or reduced oral intake should be investigated. The duration of reduced oral intake is usually short so the chance of significant vitamin deficiency is low but, if there is any doubt, supplementation with thiamine should also be given.

\section{Peripartum hyponatraemia}

Serum sodium levels fall by $4-5 \mathrm{mmol} / \mathrm{L}$ in pregnancy, for a variety of reasons. ${ }^{10}$ Systemic arterial vasodilatation causes activation of the renin-angiotensin system, leading to renal sodium reabsorption and water retention. Antidiuretic hormone $(\mathrm{ADH})$ secretion occurs at a lower serum osmolality, and causes a net gain of free water. Furthermore, during labour, oxytocin levels rise, which has the same action on the kidneys as ADH due to the structural similarity of these two posterior pituitary hormones. Pain, nausea and vomiting can also trigger $\mathrm{ADH}$ release. For these reasons, hyponatraemia is a common finding in the peripartum period. Severe hyponatraemia (serum sodium less than $120 \mathrm{mmol} / \mathrm{L}$ ) can cause lethargy and seizures.

A thorough history and examination is crucial, including assessment of fluid status, as well as bloods including plasma osmolality, when investigating hyponatraemia (Table 2). Although hyperosmotic or isosmotic hyponatraemia can occur due to uncontrolled hyperglycaemia or severe lipidaemia, the majority of cases are hypo-osmotic. $^{10}$

Severe pre-eclampsia is associated with hyponatraemia. ${ }^{11}$ In some cases, the underlying pathology is thought to be mediated by syndrome of inappropriate ADH secretion (SIADH) and hypervolaemia. ${ }^{12}$ Careful fluid assessment and fluid restriction in this situation is the mainstay of treatment. There is no safety data for the use of vasopressin antagonists (tolvaptan) in pregnancy. Intravenous fluid administration or excessive water drinking in labour, with associated high oxytocin levels, can lead to water intoxication and severe hyponatraemia. In one study, $2.5 \mathrm{~L}$ of oral and intravenous fluid administration during labour was enough to drop the serum sodium to below $130 \mathrm{mmol} / \mathrm{L}$ in $26 \%$ of women. ${ }^{13}$

Table 2. Approach to assessing peripartum

\section{hyponatraemia}

History

Symptoms Drowsiness and confusion

Past medical history Renal disease, pancreatitis, liver disease and cardiac failure

Drug history Diuretics, PPI and oxytocin

Obstetric history Duration of labour and estimated blood loss

\section{Examination}

Observations

Tachycardia and blood pressure

Fluid status JVP, peripheral oedema and crepitations on chest auscultation

\section{Investigations}

Biochemical tests Electrolytes, serum and urine osmolality, and renal profile

Fluid balance Hourly urine output

$\mathrm{JVP}=$ jugular venous pressure; $\mathrm{PPH}=$ postpartum haemorrhage; $\mathrm{PPI}=$ proton pump inhibitor. 
This is particularly prevalent in women who have experienced prolonged labour. Treatment is with fluid restriction.

As in the non-pregnant population, hypovolaemic hyponatraemia can be caused by diarrhoea and vomiting, sepsis, diuretic use, adrenal insufficiency, hypopituitarism (for example, from pituitary apoplexy) and pancreatitis. Treatment is with intravenous fluids and salt replacement.

In all cases, hypertonic sodium chloride is reserved for symptomatic cases of severe hyponatraemia, and should be administered in a high-dependency environment. Rapid correction of serum sodium can cause osmotic demyelination syndrome, to which young women are more susceptible. Extreme care should be taken while administering hypertonic sodium chloride, with regular measurements of the serum sodium levels.

Sodium and water rapidly equalises across the placenta, and a fetal sodium less than $130 \mathrm{mmol} / \mathrm{L}$ is associated with polyhydramnios, respiratory distress, jaundice and seizures. ${ }^{14}$ Fetal monitoring with cardiotocography in labour should be considered, and the neonatal team should be informed if a labouring mother is severely hyponatraemic.

\section{Conclusion}

Significant metabolic disturbances occur in pregnant women and, generally, treatment is the same as in the non-pregnant population and prompt treatment can result in rapid resolution without maternal or fetal compromise. The location of care has to be carefully considered, and depends on gestational age, medical and obstetric requirements of the woman at the time of presentation. Women may need care in a high-dependency environment, and some obstetric high-dependency units may be able to provide this. Irrespective of location, care should be delivered by a multidisciplinary team with experience in dealing with critically unwell pregnant women, and may include physicians, obstetric physicians, midwives, obstetricians, intensivists and obstetric anaesthetists.

\section{References}

1 Lain K, Catalano PM. Metabolic changes in pregnancy. Clin Obstet and Gynecol 2007;50:938-48.
2 Catalano PM, Tyzbir ED, Roman NM, Amini SB, Sims EA. Longitudinal changes in insulin release and insulin resistance in non-obese pregnant women. American J Obstet Gynecol 1991;165:1667-72.

3 Chauhan SP, Perry KG, McLaughlin BN et al. Diabetic ketoacidosis complicating pregnancy. J Perinatol 1996;16:173-5.

4 World Diabetes Foundation, Global Alliance for Women's Health. Diabetes, women and development: meeting summary, expert recommendations for policy action, conclusions and follow-up actions. Int J Gynaecol Obstet 2009;104:S46-50.

5 Sibai BM, Viteri OA. Diabetic ketoacidosis in pregnancy. Obstet Gynecol 2014;123:167-78.

6 National Institute for Health and Care Excellence. Diabetes in pregnancy: management of preconception to the postnatal period: NICE guideline [NG3]. London: NICE, 2015. www.nice.org.uk/ Guidance/NG3 [Accessed 23 June 2021].

7 National Pregnancy in Diabetes. National Pregnancy in Diabetes Annual Report 2018. London: NPID, 2019. https://digital.nhs.uk/ data-and-information/publications/statistical/national-pregnancyin-diabetes-audit/national-pregnancy-in-diabetes-annual-report2018 [Accessed 10 June 2021].

8 Owen OE, Caprio S, Reichard Jr GA et al. Ketosis of starvation: a revisit and new perspectives. Clin Endocrinol Metab 1983;12:359-79.

9 Frise C], Mackillop L, Joash K, Williamson C. Starvation ketoacidosis in pregnancy. Eur J Obstet Gynaecol Reprod Biol 2013;167:1-7.

10 Belzile M, Pouilot A, Cumyn A, Cote AM. Renal physiology and fluid and electrolyte disorders in pregnancy. Best Prac Res Clin Obstet Gynaecol 2019;57:1-14.

11 Razavi AS, Chasen ST, Gyawali R, Kalish RB. Hyponatraemia associated with preeclampsia. J Perinat Med 2017:45:467-70.

12 Powel JE, Rosenthal E, Roman A, Chasen ST, Berghella V. Preeclampsia and low sodium: a case and systematic review. Eur ] Obstet Gynaecol Reprod Biol 2020;249:14-20.

13 Moen V, Brudin L, Rundgren M, Irestedt L. Hyponatraemia complicating labour - rare or unrecognised? A prospectional observational study. BJOG 2009;116:552-61.

14 Sandhu G, Remaiyah S, Chan G, Measles I. Pathophysiology and management of preeclampsia-associated severe hyponatraemia. Am J Kidney Dis 2010;55:599-603.

Address for correspondence: Dr Naina Mohan, Women's Services, St Thomas' Hospital, Westminster Bridge Road, London SE1 7EH, UK.

Email: naina.mohan@gstt.nhs.uk 